Received: $\quad 2018.10 .30$

Accepted: 2018.11.16

Published: 2019.03 .17

\title{
Murrayanine Induces Cell Cycle Arrest, Oxidative Stress, and Inhibition of Phosphorylated p38 Expression in A549 Lung Adenocarcinoma Cells
}

Authors' Contribution: Study Design A Data Collection B Statistical Analysis C Data Interpretation D Manuscript Preparation E Literature Search F Funds Collection G

Corresponding Author: Source of support:

\author{
ABCDEF 1 Jingbao Zhang \\ BCDE 1 Yanfei Gao \\ ACDE 1 Changbo Ma \\ ACDEFG 2 Yi Wang
}

1 Department of Oncology, Jiaozhou Peoples' Hospital, Qingdao, Shandong, P.R. China

2 Department of Respiratory Medicine, Qingdao Municipal Hospital, Qingdao, Shandong, P.R. China

Background: Murrayanine is a carbazole alkaloid derived from Murraya koenigii, which has been used in traditional Chinese medicine in the treatment of cancer. This study aimed to investigate the effects of murrayanine on human lung adenocarcinoma cells in vitro and to investigate the mechanisms of its action.

Material/Methods:

Results: Murrayanine treatment resulted in significant dose-dependent inhibition of the growth of A549 cells $(p<0.05)$, with an $\mathrm{IC}_{50}$ of $9 \mu \mathrm{M}$, and arrested the cells at the G2/M phase of the cell cycle, reduced the expression of cyclin $\mathrm{D}$ and E, CDK2, 4, and 6, and increased the expression of p21 and p27. Murrayanine treatment increased apoptosis of the A549 cells and increased cleaved of caspase- 3 and caspase- 9 , and the Bax/Bcl- 2 ratio. Murrayanine treatment increased levels of reactive oxygen species (ROS), disrupted the mitochondrial membrane potential, inhibited invasion, and inhibited phosphorylation of p38 mitogen-activated protein kinase (MAPK) of the A549 cells.

Conclusions: Murrayanine induced cell cycle arrest, oxidative stress, and inhibited the expression of phosphorylated p38 in A549 adenocarcinoma cells.

MeSH Keywords: $\quad$ Apoptosis • Cell Cycle Checkpoints • Cell Migration Assays

Full-text PDF: https://www.medscimonit.com/abstract/index/idArt/913873

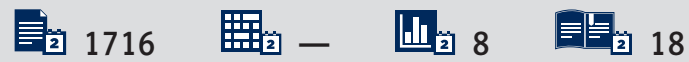




\section{Background}

Recently, there has been increasing research on drug discovery and the development of chemotherapy for the treatment of malignancy [1]. However, clinical use of many anticancer drugs is restricted by their adverse effects [2]. Naturally occurring compounds with anticancer effects have gained attention due to their low toxicity [3]. Murrayanine is a carbazole alkaloid derived from Murraya koenigii, which has been used in traditional Chinese medicine in the treatment of cancer [4]. Carbazole alkaloids have been shown to exhibit anticancer effects against a range of cancers $[5,6]$. However, the effects of murrayanine have not previously been investigated in human lung cancer.

Worldwide, lung cancer remains a leading cause of mortality from malignancy [7]. Lung cancer accounts for approximately $25 \%$ of all the cancers and results in up to $20 \%$ of cancer-related deaths [8]. The late diagnosis of lung cancer, lack of biomarkers and therapeutic targets, results in an increased need for more effective treatment [9]. Chemoresistance in lung cancer makes it even more difficult to treat [10].

Therefore the aim of this study was to investigate the effects of murrayanine on A549 human lung adenocarcinoma cells in vitro and to investigate the mechanisms of its action.

\section{Material and Methods}

\section{Cell lines and culture conditions}

The A549 human lung adenocarcinoma cell line and the normal lung fibroblast cell line, MRC-5, were obtained from the American Type Culture Collection (ATCC) (Manassas, VA, USA). The cell lines were maintained in Dulbecco's modified Eagle's medium (DMEM) containing 10\% fetal bovine serum, antibiotics (100 units/mL penicillin and $100 \mu \mathrm{g} / \mathrm{mL}$ streptomycin), and $2 \mathrm{mM}$ glutamine. The cells were cultured in an incubator at $37^{\circ} \mathrm{C}$ with $98 \%$ humidity and $5 \% \mathrm{CO}_{2}$ (Thermofisher Scientific, Waltham, MA, USA).

\section{Cell viability assay}

At around $70 \%$ confluence, the A549 and the MRC-5 cells were seeded in 96-well plates and treated with 0-200 $\mu \mathrm{M}$ of murrayanine ( $98 \%$ purity by high-performance liquid chromatography) (Sigma-Aldrich, St. Louis MO, USA). After 24 hours, the cells were incubated with MTT for $4 \mathrm{~h}$. The media was removed and the colored formazan product was solubilized by $200 \mu$ of dimethyl sulfoxide (DMSO). The viability of the A549 and the MRC -5 cells was then determined at an absorbance at $570 \mathrm{~nm}$.

\section{Apoptosis assays}

The A549 cells were grown in 6 -well plates $\left(0.6 \times 10^{6}\right.$ cells/well). Following an incubation period of around 12 hours, the cells were treated with murrayanine for $24 \mathrm{~h}$ at $37^{\circ} \mathrm{C}$. As the cells detached from the wells, $25 \mu \mathrm{l}$ of cell cultures were placed onto glass slides and stained with a solution of acridine orange and ethidium bromide, or propidium iodide (PI), or 4',6-diamidino-2-phenylindole (DAPI). The slides were then covered with a coverslip and examined with a fluorescent microscope.

\section{Cell cycle analysis}

After incubating the A549 lung cancer cells with increasing concentrations of murrayanine $(0,9,18$, and $36 \mu \mathrm{M})$ for $24 \mathrm{~h}$, the cells were washed with phosphate buffered saline (PBS). The A549 cells were stained with propidium iodide (PI) and the distribution of the cells in cell cycle phases was assessed by fluorescence-activated cell sorting (FACS) and flow cytometry.

\section{Reactive oxygen species (ROS) and mitochondrial membrane potential}

For determination of the ROS and mitochondrial membrane potential levels, the A549 cells were treated with $0,9,18$, and $36 \mu \mathrm{M}$ concentrations of murrayanine for 24 hours and then the ROS and mitochondrial membrane potential levels in the A549 cells were determined, as described previously [11].

\section{Cell invasion assays}

The murrayanine-treated A549 cells were seeded onto the Matrigel chamber $\left(1 \times 10^{5}\right.$ cells/chamber $)$ and inserted into a well of a 24-well plate, followed by the addition of FBS (10\%) to the bottom chamber. After $24 \mathrm{~h}$ of incubation, the invasive cells on the lower surface of the chamber were stained with crystal violet $(0.1 \%)$ and the cells were counted.

\section{Western blot}

The A549 cells were washed with ice-cold PBS and resuspended in a lysis buffer at $4^{\circ} \mathrm{C}$ and then incubated at $95^{\circ} \mathrm{C}$. The protein content of each cell extract was determined using the Bradford assay. Then, $40 \mu \mathrm{g}$ of protein was loaded from each sample and separated by sodium dodecyl sulfate-polyacrylamide gel electrophoresis (SDS-PAGE) before being transferred to a polyvinylidene fluoride (PVDF) membrane. The membranes were then washed with TBS and incubated in primary antibodies at $4^{\circ} \mathrm{C}$. The cells were treated with appropriate secondary antibodies and the proteins were visualized by enhanced chemiluminescence $(E C L)$ reagent. 
A

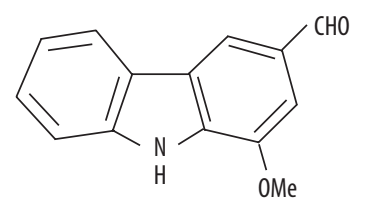

B

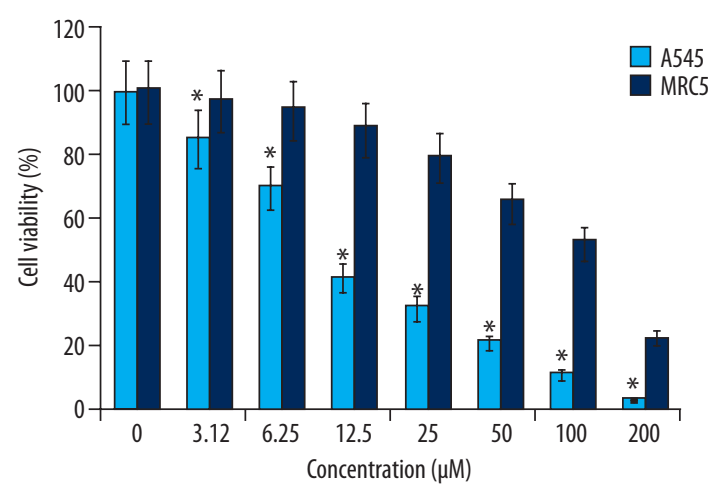

Figure 1. Murrayanine inhibited the proliferation of A549 human lung adenocarcinoma cells in vitro. (A) The chemical structure of murrayanine, a carbazole alkaloid. (B) The MTT assay shows the effects of murrayanine on the viability of the A549 lung cancer cells and MRC-5 normal lung fibroblasts. The experiments were performed in triplicate. Data are expressed as the mean $\pm S D\left({ }^{*} P<0.05\right)$
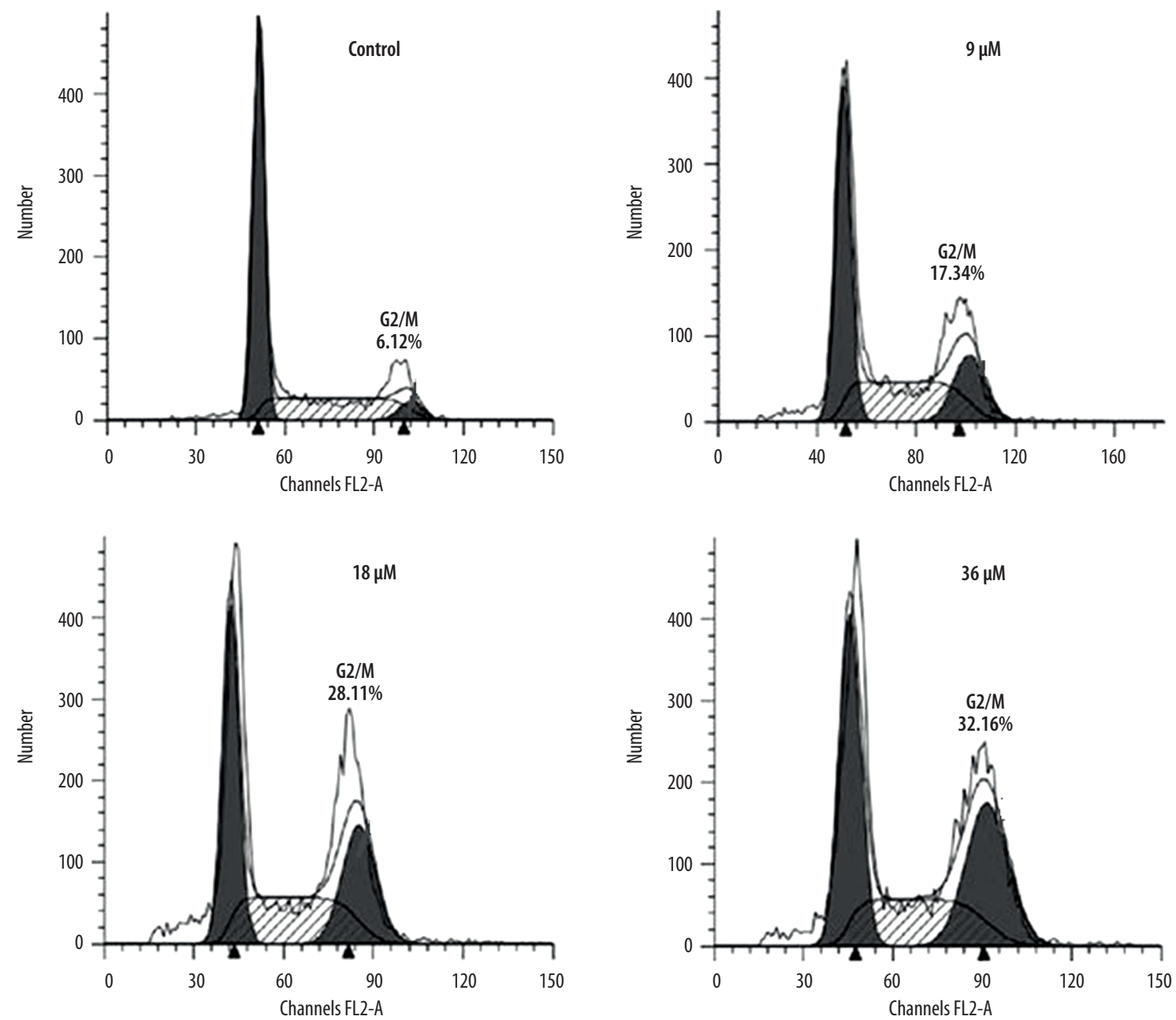

Figure 2. Flow cytometry analysis of murrayanine-treated A549 human lung adenocarcinoma cells show G2/M cell cycle arrest. The experiments were performed in triplicate. 


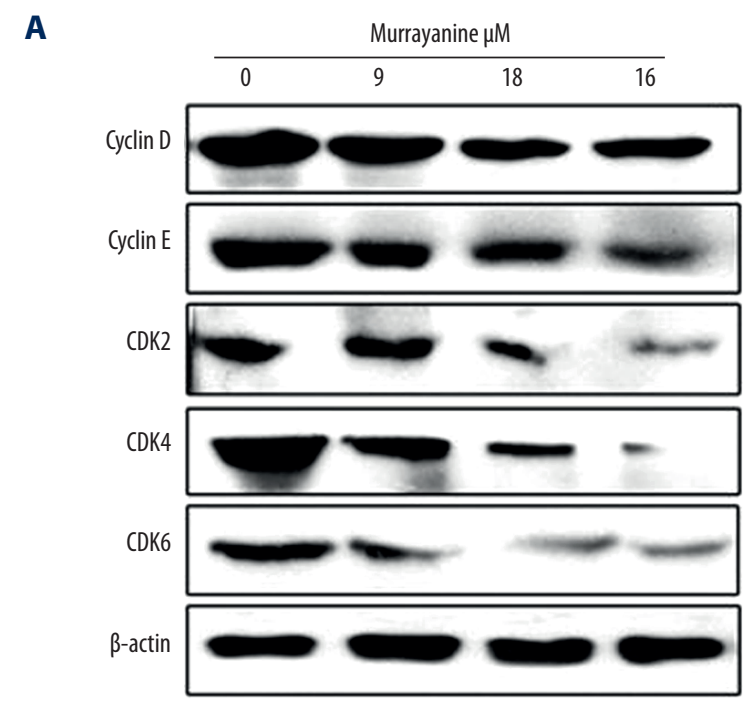

B

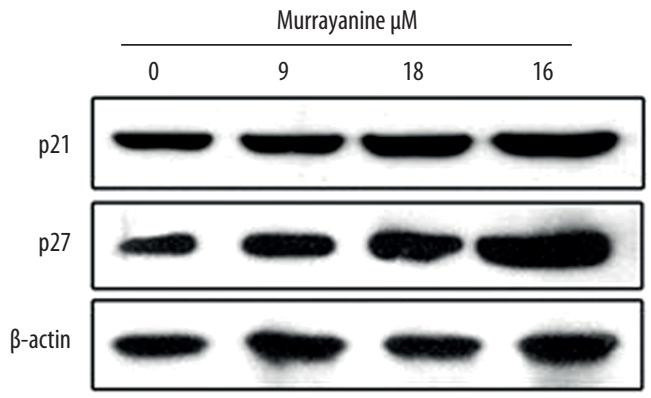

Figure 3. Western blot analysis shows the effect of murrayanine on A549 human lung adenocarcinoma cells. (A) Western blot results for cyclin D, cyclin E, CDK2, CDK4, and CDK6, at indicated concentrations. (B) Western blot results for p21 and p27, at indicated concentrations. The experiments were performed in triplicate.

\section{Statistical analysis}

Statistical analysis was performed with one-way analysis of variance (ANOVA) followed by Tukey's test using the SPSS software package, version 9.05 (IBM, Chicago, IL, USA). The values were presented as the mean $\pm \mathrm{SD}$. A P-value $<0.05$ was considered to be statistically significant.

\section{Results}

\section{Murrayanine inhibited the proliferation of A549 lung cancer cells}

The effects of murrayanine were examined on the A549 lung cancer cells and the normal MRC- 5 cells by MTT assay at concentrations ranging from 0-200 $\mu \mathrm{M}$ (Figure 1A). Murrayanine was found to inhibit the growth of the A549 cells in a concentration-dependent manner (Figure $1 \mathrm{~B}$ ). The $\mathrm{IC}_{50}$ of murrayanine treatment of the $A 549$ cells was found be at $9 \mu \mathrm{M}$. However, the effects of murrayanine treatment on the proliferation of the MRC- 5 cells were negligible. The $I C_{50}$ of murrayanine against the normal MRC-5 cells was $>100$ (Figure 1B).

\section{Murrayanine arrested the A549 cells at the G2/M checkpoint}

To understand the mechanism of action of the anti-proliferative effects of murrayanine, the distribution of the cell cycle phase in A549 cells was assessed by flow cytometry at 0,9 , 18 , and $36 \mu \mathrm{M}$ concentration of murrayanine. The percentage of G2/M phase cells increased significantly from $5.27 \%$ in the control to $33.17 \%$ at a concentration of $36 \mu \mathrm{M}$ of murrayanine (Figure 2). The arrest of the A549 cells at the G2/M phase was associated with alterations in the expression of several of the cell cycle-related proteins. The protein expression of cyclin $D$, cyclin E, CDK2, CDK4, and CDK6 were reduced following murrayanine treatment (Figure $3 \mathrm{~A}$ ), while the expression of p21 and p27 was significantly increased (Figure 3B).

\section{Murrayanine induced apoptosis of A549 cells}

The apoptosis in the murrayanine treated A549 cells was determined by using 4', 6-diamidino-2-phenylindole (DAPI), acridine orange and ethidium bromide, and propidium iodide ( $\mathrm{PI}$ ) staining. Using the three assays, the percentage of the apoptotic cells increased with increasing concentrations of murrayanine (Figure 4). Apoptosis of the murrayanine-treated A549 cells was further confirmed by examining the protein levels of apoptosis-related proteins by Western blot. Murrayanine treatment increased the cleavage of caspase- 3 and caspase- 9 in a concentration-dependent manner. Expression of Bax was increased and the expression of $\mathrm{Bcl}-2$ was decreased following murrayanine treatment (Figure 5). The reactive oxygen species (ROS) and mitochondrial membrane potential levels of murrayanine-treated A549 cells were also investigated and the levels of ROS were significantly increased (up to $195 \%$ increase) with a decrease in the mitochondrial membrane potential levels (up to 64\% decrease) (Figure 6). 

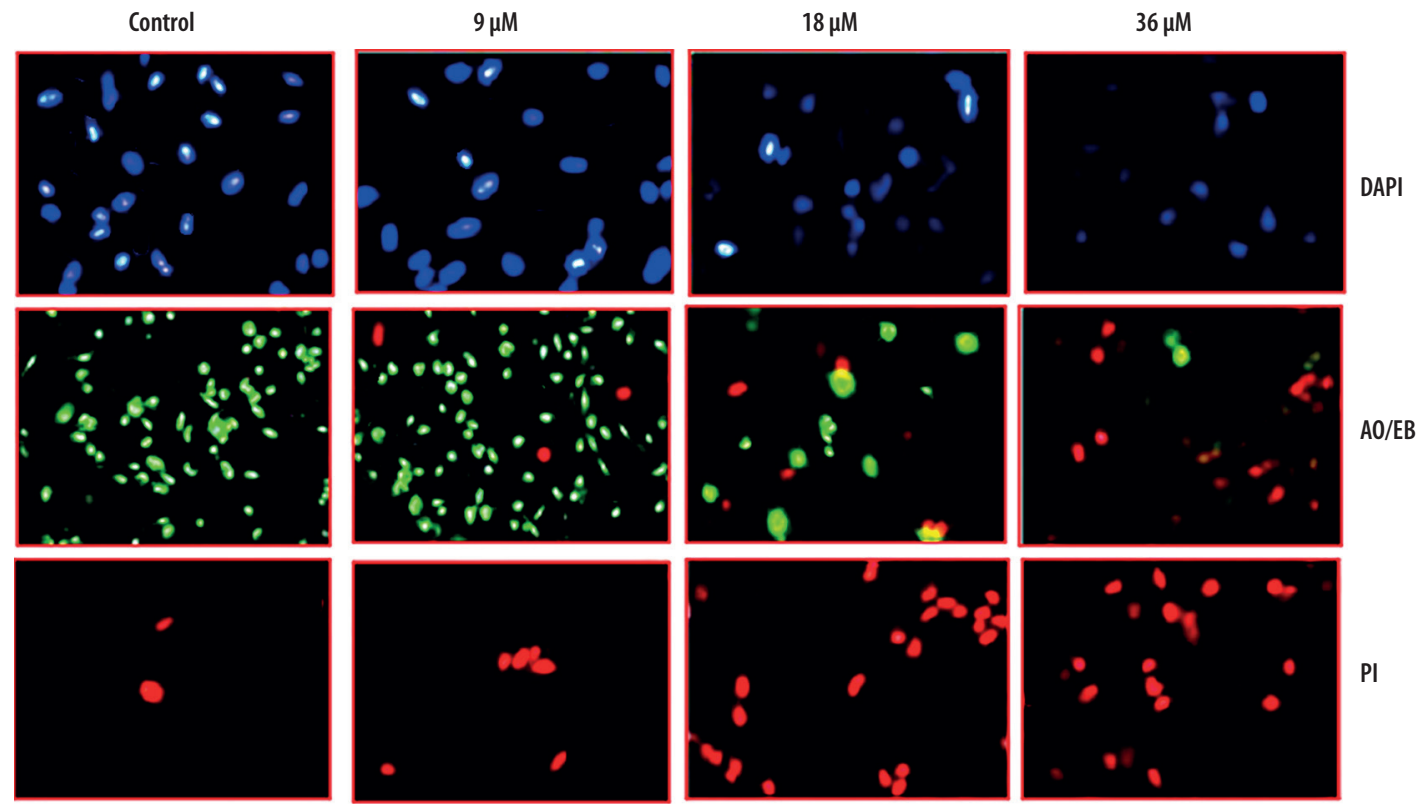

Figure 4. Fluorescence staining for apoptosis in murrayanine-treated A549 lung cancer cells. Fluorescence staining with 4', 6-diamidino-2-phenylindole (DAPI) (cell nuclei, blue), ethidium bromide, and propidium iodide (PI). The experiments were performed in triplicate.

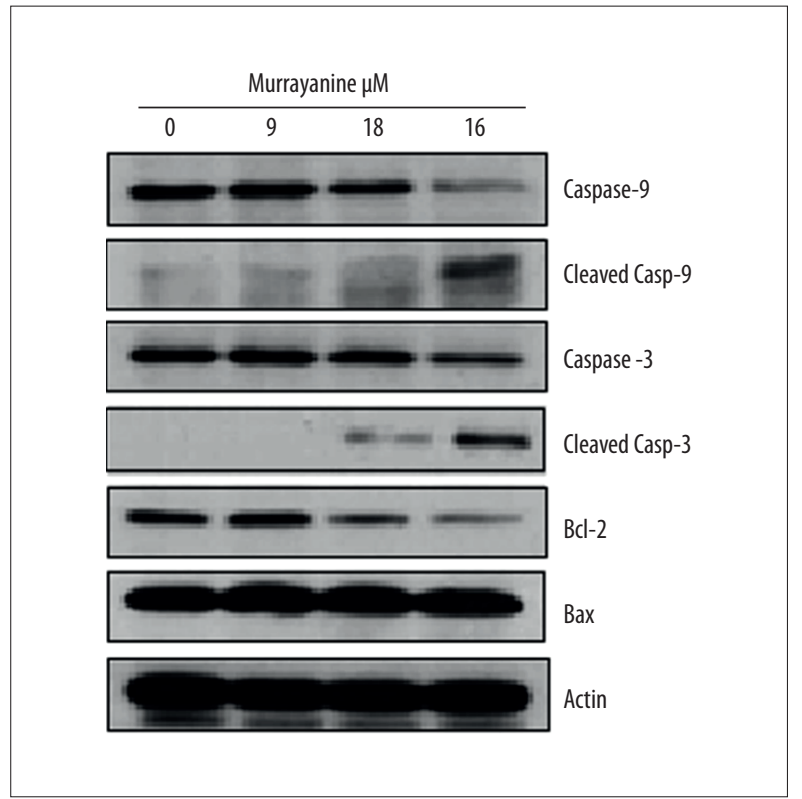

Figure 5. Western blot of apoptosis-associated proteins in A549 human lung adenocarcinoma cells. Murrayanine treatment was at the indicated concentrations. Apoptosis-related proteins include Bax, Bcl-2, caspase-3, and caspase-9, shown by Western blot. The experiments were performed in triplicate.

\section{Murrayanine inhibited the phosphorylation of p38 mitogen-activated protein kinase (MAPK) in A549 cells}

The effects of murrayanine was also examined on the phosphorylation of the p38 mitogen-activated protein kinase (MAPK) by Western blot analysis. Murrayanine inhibited the phosphorylation of p38 in a concentration-dependent manner (Figure 7).

\section{Murrayanine inhibited the invasion of A549 cells}

The effect of murrayanine treatment was also investigated on the invasion of the A549 cells by the Matrigel assay. Murrayanine inhibited the invasion of the A549 cells in a concentration-dependent manner. The invasion of the A549 cells decreased by $27 \%$ compared with the control cells at a concentration of $36 \mu \mathrm{M}$ of murrayanine (Figure 8).

\section{Discussion}

Worldwide, lung cancer is responsible for high levels of morbidity and mortality [12]. The late diagnosis, lack of diagnostic and therapeutic biomarkers, and poor response by some patients with lung cancer to chemotherapy are all hurdles to treatment [13]. In this study, the effects of murrayanine were evaluated in the A549 lung cancer cell line and the normal MRC-5 lung fibroblast cell line. Murrayanine was found to inhibit the growth of lung cancer cells by triggering $\mathrm{G} 2 / \mathrm{M}$ cell 


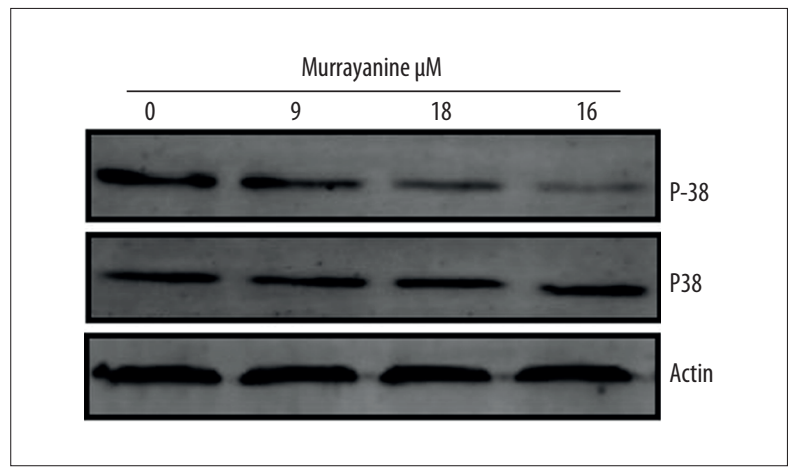

Figure 7. The effect of murrayanine treatment of A549 human lung adenocarcinoma cells on the phosphorylation of p38 as shown by Western blot analysis. The experiments were performed in triplicate.

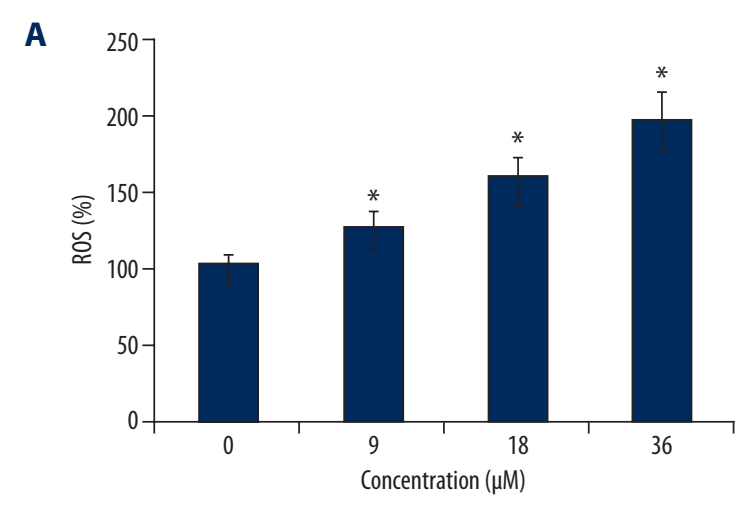

B

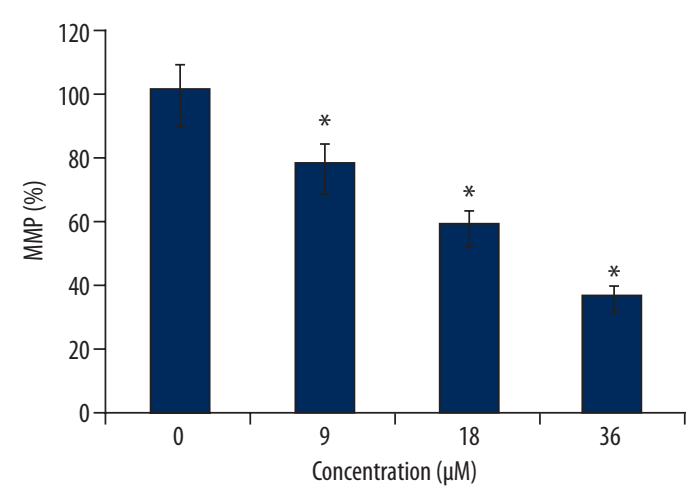

Figure 6. Reactive oxygen species (ROS) and mitochondrial membrane potential levels in A549 human lung adenocarcinoma cells treated with murrayanine. (A) Murrayanine treatment of the A549 cells resulted in a significant increase in the levels of reactive oxygen species (ROS). (B) Murrayanine treatment of the A549 cells resulted in decreased mitochondrial membrane potential levels. The experiments were performed in triplicate. The results were expressed as the mean $\pm \mathrm{SD}\left({ }^{*} \mathrm{P}<0.05\right)$

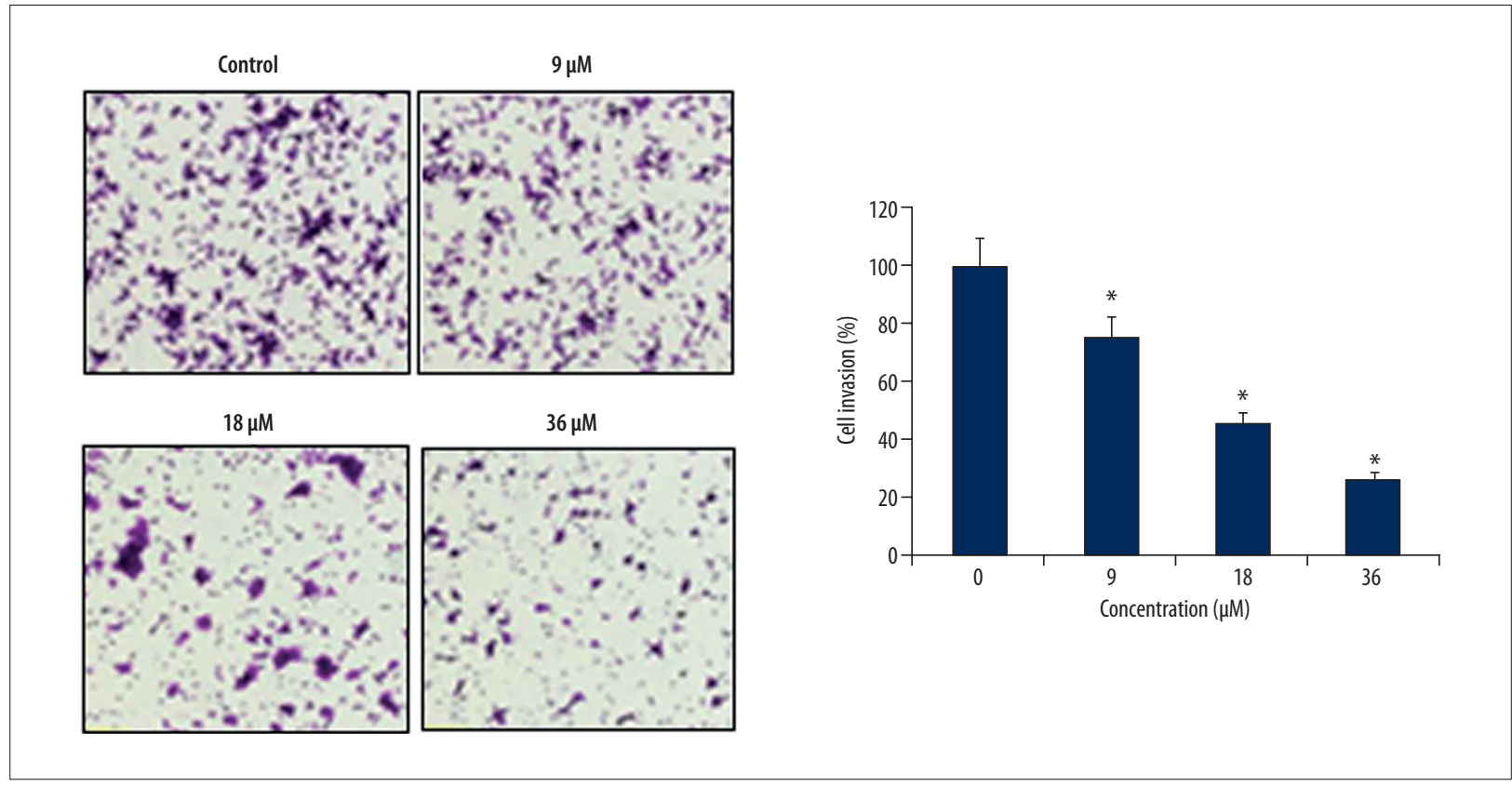

Figure 8. The concentration-dependent effect of murrayanine treatment on the invasion of the A549 lung adenocarcinoma cells. The experiments were performed in triplicate and expressed as mean $\pm S D\left({ }^{*} \mathrm{P}<0.05\right)$. 
cycle arrest. Murrayanine also induced reactive oxygen species (ROS)-mediated reduction in the mitochondrial membrane potential and was associated with apoptotic cell death of the A549 lung cancer cells, with alteration in the protein levels of Bax, Bcl-2, cleaved caspase-3, and cleaved caspase-9. Murrayanine inhibited the phosphorylation of p38 in a concentration-dependent manner. The results of the Matrigel invasion assay showed that murrayanine suppressed the migration of A549 cells.

The findings of the present study are supported by those of previous studies that have shown that several of the carbazole alkaloids inhibit the growth of cancer cells. For example, the carbazole alkaloid, mahanine, has been reported to inhibit the growth of leukemia cells [14]. Carbazole alkaloids have also been reported to inhibit the growth of cancer cells by triggering cell cycle arrest. Mahanimbine has been reported to halt the growth of pancreatic cancer cells by causing G0/G1 arrest [15]. Therefore, in the present study, the effect of murrayanine on the distribution of the cell cycle phase of the A549 cells showed that murrayanine blocked the A549 cells in the G2/M phase of the cell cycle, which was supported by reduced expression levels of cyclin B and cyclin E and upregulation of p21 and p27, which are key the regulators of cell cycle [16]. Apart from cell cycle arrest, apoptosis is a mechanism to eliminate cancer cells [15] and in this study, murrayanine was found to induce apoptosis of the A549 cells. Murrayanine also enhanced the cleavage of caspase- 3 and caspase- 9 and increased the Bax/ $\mathrm{Bcl}-2$ ratio. Increased levels of ROS and disruption of the mitochondrial membrane potential have been associated with apoptosis [17]. The findings of the present study showed that murrayanine increased ROS and decreased the mitochondrial membrane potential levels in the A549 cells, supporting cell apoptosis.

Invasion by cancer cells is required for tumor metastasis [18]. Following murrayanine treatment, the inhibition of phosphorylation of p38 mitogen-activated protein kinase (MAPK) and inhibition of invasion of the A549 cells was found in this study. Murrayanine has an antiproliferative effect and may have an effect on tumor invasion.

\section{Conclusions}

Murrayanine induced cell cycle arrest, oxidative stress, and inhibited the expression of phosphorylated p38 in A549 adenocarcinoma cells in vitro. Because of its lack of toxicity, further studies are required that include in vivo studies of the effects of murrayanine in lung cancer and other types of malignancy.

\section{Conflict of interest}

None.

\section{References:}

1. Ohnishi S, Takeda H: Herbal medicines for the treatment of cancer chemotherapy-induced side effects. Front Pharmacol, 2015; 6: 14

2. McQuade RM, Bornstein JC, Nurgali K: Anti-colorectal cancer chemotherapy-induced diarrhoea: Current treatments and side-effects. Int J Clin Med, 2014; 5: 393-406

3. Harvey AL, Edrada-Ebel R, Quinn RJ: The re-emergence of natural products for drug discovery in the genomics era. Nat Rev Drug Discov, 2015; 14(2): 111-29

4. Ng RC, Kassim NK, Yeap YS et al: Isolation of carbazole alkaloids and coumarins from Aegle marmelos and Murraya koenigii and their antioxidant properties. Sains Malaysiana, 2018; 47(8): 1749-56

5. Iman V, Mohan S, Abdelwahab SI et al: Anticancer and anti-inflammatory activities of girinimbine isolated from Murraya koenigii. Drug Des Devel Ther, 2017; 11: 103-21

6. Samanta SK, Kandimalla R, Gogoi B et al: Phytochemical portfolio and anticancer activity of Murraya koenigii and its primary active component, mahanine. Pharmacol Res, 2018; 129: 227-36

7. Didkowska J, Wojciechowska U, Mańczuk $M$ et al: Lung cancer epidemiology: Contemporary and future challenges worldwide. Ann Transl Med, 2016 4: $1-7$

8. Cheng TY, Cramb SM, Baade PD et al: The international epidemiology of lung cancer: latest trends, disparities, and tumor characteristics. J Thor Oncol, 2016; 11: 1653-71

9. Yoder LH: Lung cancer epidemiology. Medsurg Nurs, 2006; 15: 171-74

10. Miller KD, Siegel RL, Lin CC et al: Cancer treatment and survivorship statistics, 2016. Cancer J Clin, 2016; 66: 271-89

11. Hua F, Li CH, Chen XG et al: Daidzein exerts anticancer activity towards SKOV3 human ovarian cancer cells by inducing apoptosis and cell cycle arrest, and inhibiting the Raf/MEK/ERK cascade. Int J Mol Med, 2018; 41(6): 3485-92

12. Davis JN, Medbery C, Sharma S et al: Stereotactic body radiotherapy for centrally located early-stage non-small cell lung cancer or lung metastases from the RSSearch ${ }^{\circledast}$ patient registry. Radiat Oncol, 2015; 10: 113

13. Borghaei $H$, Paz-Ares L, Horn L et al: Nivolumab versus docetaxel in advanced nonsquamous non-small-cell lung cancer. N Engl J Med, 2015 373(17): 1627-39

14. Bhattacharya K, Samanta SK, Tripathi R et al: Apoptotic effects of mahanine on human leukemic cells are mediated through crosstalk between Apo-1/Fas signaling and the Bid protein and via mitochondrial pathways. Biochem Pharmacol, 2010; 79(3): 361-72

15. Pei C, He Q, Liang S, Gong X: Mahanimbine exerts anticancer effects on human pancreatic cancer cells by triggering cell cycle arrest, apoptosis, and modulation of AKT/mammalian target of rapamycin (mTOR) and signal transducer and activator of transcription 3 (STAT3) signalling pathways. Med Sci Monit, 2018; 24: 6975-83

16. Chang F, Steelman LS, Shelton JG et al: Regulation of cell cycle progression and apoptosis by the Ras/Raf/MEK/ERK pathway. Int J Oncol, 2003; 22(3): 469-80

17. Gu ZF, Zhang ZT, Wang J et al: Icariin exerts inhibitory effects on the growth and metastasis of KYSE70 human esophageal carcinoma cells via PI3K/AKT and STAT3 pathways. Environ Toxicol Pharmacol, 2017; 54: 7-13

18. Jin L, Chun J, Pan C et al: Phosphorylation-mediated activation of LDHA promotes cancer cell invasion and tumour metastasis. Oncogene, 2017 36(27): 3797-806 